\title{
The homogeneous marginal utility of income assumption
}

Citation for published version (APA):

Demuynck, T. (2015). The homogeneous marginal utility of income assumption. Maastricht University, Graduate School of Business and Economics. GSBE Research Memoranda No. 013 https://doi.org/10.26481/umagsb.2015013

Document status and date:

Published: 01/01/2015

DOI:

10.26481/umagsb.2015013

Document Version:

Publisher's PDF, also known as Version of record

\section{Please check the document version of this publication:}

- A submitted manuscript is the version of the article upon submission and before peer-review. There can be important differences between the submitted version and the official published version of record.

People interested in the research are advised to contact the author for the final version of the publication, or visit the DOI to the publisher's website.

- The final author version and the galley proof are versions of the publication after peer review.

- The final published version features the final layout of the paper including the volume, issue and page numbers.

Link to publication

\footnotetext{
General rights rights.

- You may freely distribute the URL identifying the publication in the public portal. please follow below link for the End User Agreement:

www.umlib.nl/taverne-license

Take down policy

If you believe that this document breaches copyright please contact us at:

repository@maastrichtuniversity.nl

providing details and we will investigate your claim.
}

Copyright and moral rights for the publications made accessible in the public portal are retained by the authors and/or other copyright owners and it is a condition of accessing publications that users recognise and abide by the legal requirements associated with these

- Users may download and print one copy of any publication from the public portal for the purpose of private study or research.

- You may not further distribute the material or use it for any profit-making activity or commercial gain

If the publication is distributed under the terms of Article $25 \mathrm{fa}$ of the Dutch Copyright Act, indicated by the "Taverne" license above, 


\section{Maastricht University}

Thomas Demuynck

The homogeneous marginal utility of income assumption

$\mathrm{RM} / 15 / 013$

\section{GSBE}

Maastricht University School of Business and Economics

Graduate School of Business and Economics

P.O Box 616

NL- 6200 MD Maastricht

The Netherlands 


\title{
The homogeneous marginal utility of income assumption
}

\author{
Thomas Demuynck
}

\begin{abstract}
We develop a test to verify if every agent from a population of heterogeneous consumers has the same marginal utility of income function. This homogeneous marginal utility of income assumption is often (implicitly) used in applied demand studies because it has nice aggregation properties and facilitates welfare analysis. If a dataset satisfies our test, we can further identify the common marginal utility of income function. We apply our results using a US cross sectional dataset on food consumption.
\end{abstract}

Keywords: Marginal utility of income, aggregation, nonparametric JEL code: C12, C14, D12, D60

\section{Introduction}

Whether individual indirect utility functions aggregate into an indirect utility function for a representative consumer depends crucially on the marginal utility of income functions. In order to see this, consider a setting with $n$ agents $^{1}$ and let $v_{j}(\mathbf{p}, x)$ be the indirect utility function of agent $j=1, \ldots, n$ which gives the maximal utility for $j$ obtainable at prices $\mathbf{p}$ and income $x$. Denote by $V(\mathbf{p}, x)$ the average indirect utility function over all $n$ agents,

$$
V(\mathbf{p}, x)=\frac{1}{n} \sum_{j=1}^{n} v_{j}(\mathbf{p}, x) .
$$

Notice that we aggregate conditional on some level of income which is different from how aggregation is typically performed in representative consumer settings. If $q_{j}(\mathbf{p}, x)$ denotes the Marshallian demand of agent $j$ for good $q$, then the mean demand function for good $q$ is defined by,

$$
\bar{q}(\mathbf{p}, x)=\frac{1}{n} \sum_{j=1}^{n} q_{j}(\mathbf{p}, x) .
$$

\footnotetext{
${ }^{*}$ Maastricht University. Tongersestraat 53, 6711 LM Maastricht, Netherlands, email: t.demuynck@maastrichtuniversity.nl

${ }^{1}$ This considers the case where there are only a finite number of agents, but the setting can be generalized to a continuum of consumers as will be the case in the next section.
} 
Lewbel (2001) calls this the statistical demand function, but we follow Schlee (2007) and use the term 'mean demand function'. Using Roy's identity, we can express this demand function in terms of derivatives of the indirect utility functions of the agents,

$$
\bar{q}(\mathbf{p}, x)=\frac{1}{n} \sum_{j=1}^{n}-\frac{\frac{\partial v_{j}(\mathbf{p}, x)}{\partial p_{q}}}{\frac{\partial v_{j}(\mathbf{p}, x)}{\partial x}} .
$$

On the other hand, the demand function for the representative agent with utility function $V(\mathbf{p}, x)$, is given by,

$$
Q(\mathbf{p}, x)=\frac{1}{n} \frac{\sum_{j=1}^{n}-\frac{\partial v_{j}(\mathbf{p}, x)}{\partial p_{q}}}{\sum_{j=1}^{n} \frac{\partial v_{j}(\mathbf{p}, x)}{\partial x}} .
$$

The two demand functions $Q(\mathbf{p}, x)$ and $\bar{q}(\mathbf{p}, x)$ will coincide if and only if the marginal utility of income functions, $\partial v_{j}(\mathbf{p}, x) / \partial x$, are identical for all agents $j=1, \ldots, n .^{2}$ We call this the Homogeneous Marginal Utility of Income assumption (HMUI). By integrating out the indirect utility function, we obtain that the HMUI assumption requires the existence of agent specific functions $A_{j}$ and a function $B$ such that,

$$
v_{j}(\mathbf{p}, x)=A_{j}(\mathbf{p})+B(\mathbf{p}, x) .
$$

The key feature of this utility function is that income only enters via the function $B$ which is the same for all consumers. We refer to Lewbel (2001) and Schlee (2007) for a detailed discussion of this family of indirect utility functions.

The HMUI assumption is frequently imposed on many functional forms in the parametric demand literature (like the Gorman polar form (Gorman, 1961) or the Almost Ideal Demand System (Deaton and Muellbauer, 1980)). It is easy to see that the HMUI assumption implies that individual demand functions can be expresses as the sum of the average demand function, $Q(\mathbf{p}, x)$, which satisfies all regularity properties of an individual demand function (i.e. homogeneity, adding up, Slutsky symmetry and negativity), and an individual heterogeneity term that has zero mean. Given this, the representative demand function $Q(\mathbf{p}, x)$ can be estimated using a parametric or nonparametric conditional mean estimator. Given that this demand function satisfies all regularity assumptions it can also be used to conduct welfare analysis, e.g. computation of the deadweight loss of proposed tax changes or the computation of (average) equivalent and compensating variation. We refer to Hausman and Newey (1995), Blundell, Browning, and Crawford (2003) and Blundell, Horowitz, and Parey (2012) for such applications. Schlee (2007) showed that if the HMUI assumption is satisfied and if the marginal utility of income decreases with income (which is a reasonable assumption), then the compensating variation obtained from using the mean demand function, $Q(\mathbf{p}, x)$, will be a lowerbound for the mean of the agents' compensating variation.

\footnotetext{
${ }^{2}$ The necessary part of this result requires that $Q(\mathbf{p}, x)$ equals $\bar{q}(\mathbf{p}, x)$ for all distributions of agents and agents have different demand functions.
} 
An additional benefit of the HMUI assumption is that it allows for interpersonal comparison of utility. For example, if we redistribute one dollar from an individual with income $x$ towards an individual with income $y$, then aggregate utility changes by $\frac{\partial B(\mathbf{p}, y)}{\partial y}-\frac{\partial B(\mathbf{p}, x)}{\partial x}$, which is independent of the identities of the two agents. In this sense, the HMUI assumption greatly simplifies cost benefit analysis.

Contribution The main contribution of this paper is to provide a test for the HMUI assumption. We provide two sets of tests. The first is based on the conditional quantiles of the consumption share function. The second is based on the values of the conditional moments. The tests are obtained using an over-identification result on the values on the marginal utility of income function (given that the HMUI assumption holds). As such, if our test is not rejected, it becomes possible to identify this common marginal utility of income function.

The issue of measuring the marginal utility of income is an old problem which dates back to the contributions of Frisch (1932), Samuelson (1937), Vickrey (1945) and Morgan (1945). More recent studies try to measure the marginal utility of income using data from happiness studies (Layard, Nickell, and Mayraz, 2008). In this paper, we show that the marginal utility of income function is easily identified if the HMUI assumption holds. Interestingly, our test and identification result only require data on the consumption of a single good or group of goods and the 'disposable' income for a single cross section of consumers. In this sense, there is no need for multiple cross sections, panel data or consumption data on multiple goods. On the other hand, if observations on multiple goods are available, additional testable restrictions can be obtained.

We implement our test using the 2007 wave of the Consumer Expenditure Survey (CEX), a US consumer budget survey. We focus our analysis on the consumption of food. Based on this data set, we find that we cannot reject the HMUI assumption at the $90 \%$ confidence level. Next, we estimate the marginal utility of income function. This allows us to make interpersonal comparisons of utility for agents that are positioned at different places in the income distribution. According to our estimates, aggregate welfare increases equally when we either given one dollar to an agent at the median of the income distribution, 0.61 dollars to someone at the first decile, or 1.45 dollars to someone at the 9th decile.

Overview Section 2 contains the framework and the main testability and identification results. Section 3 presents our estimation procedure and describes our statistical test for the HMUI assumption. Section 4 contains the application and section 5 concludes.

\section{Testable implications of the HMUI assumption}

We consider a probability space $(J, \mathcal{J}, \mathrm{P})$ of agents where agent $j \in J$ has a continuously differentiable indirect utility function $v_{j}(\mathbf{p}, x)$ that satisfies the HMUI assumption, i.e.,

$$
v_{j}(\mathbf{p}, x)=A_{j}(\mathbf{p})+B(\mathbf{p}, x) .
$$

Let $\mathbf{q}_{S}$ be a subset of goods with price vector $\mathbf{p}_{S}$. The share of these goods as a fraction of the total expenditure $x$ is given by $s=\frac{\mathbf{p}_{S}^{\prime} \mathbf{q}_{S}}{x}$. Using Roy's identity, we see that the share function for the 
goods in $S$ for agent $j$ has the following functional structure,

$$
s_{j}(\mathbf{p}, x)=-\frac{a_{j}(\mathbf{p})+b(\mathbf{p}, x)}{x \beta(\mathbf{p}, x)},
$$

where $a_{j}(\mathbf{p})=\mathbf{p}_{S}^{\prime} \nabla_{\mathbf{p}_{S}} A_{j}(\mathbf{p}), b(\mathbf{p}, x)=\mathbf{p}_{S}^{\prime} \nabla_{p_{S}} B(\mathbf{p}, x)$ and $\beta(\mathbf{p}, x)=\partial B(\mathbf{p}, x) / \partial x$. We take this functional form as a starting point to develop our test. Observe that $\beta(\mathbf{p}, x)$, being the marginal utility of income, is strictly positive.

We consider the setting where we have a single cross section with fixed prices $\mathbf{p}$ for all goods and we observe the consumption share of the goods in $S$, together with the disposable income for a large number of agents. This allows us to identify the joint distribution of $\left(s_{j}, x_{j}\right)$ where $s_{j}=s_{j}\left(\mathbf{p}, x_{j}\right)$, i.e. $s_{j}$ is the consumption share of the goods in $S$ for agent $j$ with income $x_{j}$. Given that we restrict ourselves to a single cross section, with identical prices for all consumers, we omit from now on the dependency on prices and simply write $s_{j}(x)$ instead of $s_{j}(\mathbf{p}, x)$, i.e. $s_{j}(x)$ is the Engel share curve of consumer $j$.

If we observe in addition some individual characteristics, like household size, education level, marital status, age, etc. it is possible to take these into account by performing the entire analysis conditional on some value of these characteristics. In addition, this would allow us to make both functions $A_{j}$ and $B$ dependent on these observable characteristics. For ease of notation, however, we omit such observable characteristics although we will control for household composition and some other characteristics in the empirical application.

We assume that $a_{j}$ and $x_{j}$ are independently distributed. In particular, for any level of income $x$ and any value $a \in \mathbb{R}$, we assume that,

$$
\operatorname{Pr}\left(a_{j} \leq a \mid x_{j}=x\right)=\operatorname{Pr}\left(a_{j} \leq a\right) .
$$

Observe that this implies that,

$$
\operatorname{Pr}\left(s_{j} \leq s \mid x_{j}=x\right)=\operatorname{Pr}\left(s_{j}(x) \leq s\right) .
$$

The term $a_{j}$ captures the unobserved preference heterogeneity in the population. In this sense, above condition states that the distribution of this heterogeneity component is independent of the level of expenditure. This is not an innocuous assumption but similar assumptions are frequently used in the literature. ${ }^{3}$ Again, if we have observations on additional household characteristics, the independence assumption can be relaxed to independence conditional on the value of these characteristics. The independence assumption also rules out endogeneity of the expenditure level. In principle, endogeneity of the expenditure level could be allowed for by using nonparametric IV or control function approaches. We come back to this issue in the next section.

Finally, we assume that the cumulative distribution function of $-a_{j}(\mathbf{p})$ is strictly increasing on its support.

\footnotetext{
${ }^{3}$ See for example, Lewbel (2001, equation 4), Hausman and Newey (2013, Assumption 1) and Blundell, Kristensen, and Matzkin (2014, Assumption A.1).
} 
Let $\bar{s}(x)$ be the mean Engel share curve, conditional on the expenditure level $x$,

$$
\begin{aligned}
\bar{s}(x) & =\int s_{j} \mathrm{dP}\left(s_{j} \mid x_{j}=x\right), \\
& =-\frac{\int a_{j} \mathrm{dP}\left(a_{j} \mid x_{j}=x\right)+b(x)}{x \beta(x)}, \\
& =-\frac{\int a_{j} \mathrm{dP}\left(a_{j}\right)+b(x)}{x \beta(x)} \equiv-\frac{\bar{a}+b(x)}{x \beta(x)} .
\end{aligned}
$$

\subsection{Testable implications from conditional quantiles}

Let $z_{\pi}(x)$ be the $\pi$-th quantile of the distribution of the random variable $\left(s_{j}-\bar{s}\left(x_{j}\right)\right)$ conditional on the expenditure level $x_{j}=x$, (i.e., the $\pi$-th conditional quantile of the error $s_{j}-E\left(s_{j} \mid x_{j}\right)$ ),

$$
\operatorname{Pr}\left(s_{j}-\bar{s}\left(x_{j}\right) \leq z_{\pi}(x) \mid x_{j}=x\right)=\pi .
$$

The value of $z_{\pi}(x)$ is identified from the joint distribution of $\left(s_{j}, x_{j}\right)$. The following proposition shows that for the HMUI assumption to hold, the ratio of $z_{\pi}(x) x$ and $z_{\pi}(y) y$, for two different incomes $x$ and $y$, should be independent of $\pi$.

Proposition 1. Assume that the share functions $s_{j}($.$) are of the form (1), then for all income levels$ $x$ and $y$,

$$
z_{\pi}(x) x \beta(x)=z_{\pi}(y) \text { y } \beta(y) .
$$

in particular, if $z_{\pi}(y) \neq 0$, then

$$
\frac{z_{\pi}(x) x}{z_{\pi}(y) y}=\frac{\beta(y)}{\beta(x)}
$$

Proof. If the demand functions are of the form (1), then we have that,

$$
\begin{aligned}
& \operatorname{Pr}\left(s_{j}-\bar{s}\left(x_{j}\right) \leq z_{\pi}(x) \mid x_{j}=x\right)=\pi, \\
\Longleftrightarrow & \operatorname{Pr}\left(-a_{j}+\bar{a} \leq z_{\pi}(x) \beta\left(x_{j}\right) x_{j} \mid x_{j}=x\right)=\pi, \\
\Longleftrightarrow & \operatorname{Pr}\left(-a_{j} \leq z_{\pi}(x) \beta(x) x-\bar{a}\right)=\pi .
\end{aligned}
$$

Here the last line follows from the independency assumption. Performing the same derivation at the income level $y$ gives,

$$
\begin{aligned}
& \operatorname{Pr}\left(s_{j}-\bar{s}\left(x_{j}\right) \leq z_{\pi}(y) \mid x_{j}=y\right)=\pi, \\
\Longleftrightarrow & \operatorname{Pr}\left(-a_{j} \leq z_{\pi}(y) \beta(y) y-\bar{a}\right)=\pi,
\end{aligned}
$$

Equating these two expressions, we obtain that $z_{\pi}(x) \beta(x) x=z_{\pi}(y) \beta(y) y$ or equivalently (if $\left.z_{\pi}(y) \neq 0\right)$

$$
\frac{z_{\pi}(x) x}{z_{\pi}(y) y}=\frac{\beta(y)}{\beta(x)}
$$


Condition (3) shows two things. First of all, the right hand side is independent of $\pi$, which means that the left hand side should also be independent of $\pi$. This provides us with a test for the hypothesis that the HMUI assumption is satisfied. In fact, the right hand side is also independent of the particular subset of goods under consideration. Given this, if we have observations on the consumption of more than one good or group of goods, this would yield an additional testable implication. Second, if it is indeed the case that the left hand side is independent of $\pi$, then condition (3) shows how to identify the value of $\beta(y)$, up to a normalization, i.e. the marginal utility of income function is identified.

Proposition 1 shows that condition (3) is a necessary condition if every individual has a share function of the form (1). The following proposition shows that the reverse also holds.

Proposition 2. Assume that condition (2) is satisfied given the joint distribution of $\left(s_{j}, x_{j}\right)$ at all income levels $x$ and $y$. Then there is a probability space $\left(\Pi, \mathcal{J}^{\prime}, P^{\prime}\right)$ of agents and for each $\pi \in \Pi$, there is a share functions $s_{\pi}(x)$ of the form (1) and an income $x_{\pi}$ such that the joint distribution of $\left(s_{\pi}\left(x_{\pi}\right), x_{\pi}\right)$ is the same as the observed joint distribution $\left(s_{j}, x_{j}\right)$.

Proof. Consider the probability space $\left(\Pi, \mathcal{J}^{\prime}, P^{\prime}\right)$ where $\Pi$ is the unit interval and $P^{\prime}$ is the uniform distribution on this interval. For an agent $\pi \in[0,1]$. Define the share function of consumer $\pi$, denoted by $s_{\pi}(x)$ as the $\pi$ th quantile share function corresponding to the distribution of $s_{j}$ conditional on $x_{j}=x$, i.e.

$$
\operatorname{Pr}\left(s_{j} \leq s_{\pi}(x) \mid x_{j}=x\right)=\pi .
$$

By definition $s_{\pi}(x)=\left(z_{\pi}(x)+\bar{s}(x)\right)$. Fix an arbitrary income $\bar{y}$. Then if equation (2) is satisfied, we know that,

$$
s_{\pi}(x)=\left(z_{\pi}(x)+\bar{s}(x)\right)=\frac{z_{\pi}(\bar{y}) \beta(\bar{y}) \bar{y}}{x \beta(x)}+\frac{\bar{s}(x)}{x \beta(x)} .
$$

Defining $-a_{\pi}=z_{\pi}(\bar{y}) \beta(\bar{y}) \bar{y}$ and $-b(x)=\bar{s}(x)$ shows that $s_{\pi}(x)$ can be written in the form (1).

Next, set the marginal distribution of $x_{\pi}$ equal the marginal distribution of $x_{j}$ (say $F($.$) ) and$ let $x_{\pi}$ be independently distributed of $a_{\pi}$. Let $I($.$) be the indicator function. Then,$

$$
\begin{aligned}
\operatorname{Pr}\left(s_{\pi}\left(x_{\pi}\right) \leq s \text { and } x_{\pi} \leq y\right) & =\int_{0}^{y} \int I\left(s_{\pi}\left(x_{\pi}\right) \leq s\right) \mathrm{dP}\left(\pi \mid x_{\pi}=x\right) \mathrm{d} F(x), \\
& =\int_{0}^{y} \int I\left(s_{\pi}(x) \leq s\right) \mathrm{dP}(\pi) \mathrm{d} F(x), \\
& =\int_{0}^{y} \int_{0}^{1} I\left(s_{\pi}(x) \leq s\right) \mathrm{d} \pi \mathrm{d} F(x) . \\
& =\int_{0}^{y} \int I\left(s_{j}(x) \leq s\right) \mathrm{dP}(j) \mathrm{d} F(x), \\
& =\int_{0}^{y} \int I\left(s_{j}\left(x_{j}\right) \leq s\right) \mathrm{dP}\left(j \mid x_{j}=x\right) \mathrm{d} F(x), \\
& =\int_{0}^{y} \int I\left(s_{j} \leq s\right) \mathrm{dP}\left(s_{j} \mid x_{j}=x\right) \mathrm{d} F(x), \\
& =\operatorname{Pr}\left(s_{j}\left(x_{j}\right) \leq s \text { and } x_{j} \leq y\right) .
\end{aligned}
$$


This shows that the joint distribution of $\left(s_{j}, x_{j}\right)$ is the same as the distribution of $\left(s_{\pi}\left(x_{\pi}\right), x_{\pi}\right)$.

Proposition 2 shows that (3) is not only a necessary condition for (1) but also sufficient for the existence of a population with demand share functions that satisfy (1) and generate the observed demand distribution. Given this, we base our test of the HMUI assumption on equation (3). However, one caveat is in order. Notice that the identification fails if $z_{\pi}(y)=0$. This would occur if, for example, $\pi=0.5$ and the conditional distribution of $s_{j}$ given $x_{j}=x$ is symmetric around $\bar{s}(x)$. As such, we should focus only on values of $\pi$ for which $z_{\pi}(y)$ is significantly different from zero.

\subsection{Testable implications from conditional moments}

The procedure above developed testable implications by using the conditional quantiles of the variable $s_{j}-\bar{s}\left(x_{j}\right)$. A similar result can be obtained by using the conditional moments of the variable $s_{j}$. The variance of $s_{j}$ conditional on $x_{j}=x$ (if it exists) is defined by,

$$
\begin{aligned}
\mu^{2}(x) & =\int\left(s_{j}-\bar{s}\left(x_{j}\right)\right)^{2} \mathrm{dP}\left(s_{j} \mid x_{j}=x\right), \\
& =\int\left(\frac{-a_{j}+\bar{a}}{\beta\left(x_{j}\right) x_{j}}\right)^{2} \mathrm{dP}\left(a_{j} \mid x_{j}=x\right), \\
& =\frac{1}{\beta(x)^{2} x^{2}} \int\left(a_{j}-\bar{a}\right)^{2} \operatorname{dP}\left(a_{j}\right)
\end{aligned}
$$

From this, we obtain that for two different income levels $x$ and $y$,

$$
\sqrt{\frac{\mu^{2}(x)}{\mu^{2}(y)}} \frac{x}{y}=\frac{\beta(y)}{\beta(x)} .
$$

More general, if the the $m$-th conditional moments $\mu^{m}(x)=\int_{j}\left(s_{j}-\bar{s}\left(x_{j}\right)\right)^{m} \mathrm{dP}\left(s_{j} \mid x_{j}=x\right)$ exist, we have the following result.

Proposition 3. Assume that the share functions $s_{j}($.$) are of the form (1), then for all income levels$ $x$ and $y$,

$$
\left(\mu^{m}(x)\right)^{1 / m} x \beta(x)=\left(\mu^{m}(y)\right)^{1 / m} y \beta(y) .
$$

in particular, if $\mu^{m}(y) \neq 0$, then

$$
\left(\frac{\mu^{m}(x)}{\mu^{m}(y)}\right)^{1 / m} \frac{x}{y}=\frac{\beta(y)}{\beta(x)} .
$$

We see that the right hand side of equation (5) is independent of $m$ which gives another set of testable implications. Moreover, it gives us another means to identify the marginal utility of income function. Caution should be taken when $\mu^{m}(y)$ is (close to) zero, which can happen if the conditional distribution is symmetric and $m$ is odd. Given this, we focus in our empirical analysis on the even moments. 


\section{Implementation}

We estimate the conditional quantiles and moments in two steps. Consider an iid sample $j=$ $1, \ldots, n$ of shares and incomes $\left(s_{j}, x_{j}\right)$. First, we nonparametrically estimate the mean consumption share function $\bar{s}\left(x_{i}\right)$. We do this using a (leave one out) local linear kernel regression of the consumption shares $s_{j}$ on the log of expenditure, $\ln \left(x_{j}\right)$. In particular, we define, $\hat{s}(x)$ by,

$$
\hat{s}\left(x_{i}\right)=\underset{a_{0}}{\operatorname{argmin}} \min _{b_{0}} \sum_{j \neq i}\left[s_{j}-a_{0}-b_{0}\left(\ln \left(x_{i}\right)-\ln \left(x_{j}\right)\right)\right]^{2} k\left(\frac{\ln \left(x_{j}\right)-\ln \left(x_{i}\right)}{h_{n}}\right),
$$

where $k($.$) is a symmetric kernel and h_{n}$ is the bandwidth. In practice, we use the Gaussian kernel and choose $h_{n}$ using cross-validation. If $h \rightarrow 0$ and $n h_{n} \rightarrow \infty$ for $n \rightarrow \infty$, we know that the estimator $\hat{s}\left(x_{i}\right)$ consistently estimates $\bar{s}\left(x_{i}\right)$. Under suitable conditions (see Li and Racine (2007, section 2.4)) the random variable $\sqrt{n h_{n}}\left(\hat{s}\left(x_{i}\right)-\bar{s}\left(x_{i}\right)\right)$ has an asymptotic normal distribution.

Next, we estimate $\operatorname{Pr}\left(s_{j}-\hat{s}\left(x_{j}\right) \leq z \mid x_{j}=x\right)$ by using a smoothed nonparametric conditional cdf estimator,

$$
\underset{a_{1}}{\operatorname{argmin}} \min _{b_{1}} \sum_{j}\left[K\left(\frac{z-s_{j}+\hat{s}\left(x_{j}\right)}{h_{2}}\right)-a_{1}-b_{1}\left(\ln \left(x_{j}\right)-\ln (x)\right)\right]^{2} k\left(\frac{\ln \left(x_{j}\right)-\ln (x)}{h_{n}}\right)
$$

where $K(y)=\int_{-\infty}^{y} k(\psi) d \psi$ and $h_{2}$ is a suitable chosen bandwidth of the order $o\left(h_{n}\right)$. Finally, we estimate $z_{\pi}(x)$ as the nonparametric quantile estimator, $\hat{z}_{\pi}(x)$ of this local linear kernel estimator (see Li and Racine (2007, section 6.3)). Other estimators for the conditional cdf and quantiles are possible but provide very similar results. The derivation of the asymptotic properties of $\hat{z}_{\pi}(x)$ is made difficult by the fact that the mean share function $\bar{s}\left(x_{j}\right)$ is replaced by the estimate $\hat{s}\left(x_{j}\right)$. However, using a proof which is very similar to the one of Zhou and Zhu (2014, Theorems 1 and 2 ) it can be shown that the estimator satisfies the so called adaptiveness property which means that substituting the true mean share function $\bar{s}\left(x_{j}\right)$ by its estimate $\hat{s}\left(x_{j}\right)$ does not change the consistency of $\hat{z}_{\pi}(x)$ or asymptotic normality of $\sqrt{n h_{n}}\left(\hat{z}_{\pi}(x)-z_{\pi}(x)\right)$.

The conditional moments $\mu(x)^{m}$ are estimated as the solution of $a_{2}$ in the following minimization problem.

$$
\underset{a_{2}}{\operatorname{argmin}} \min _{b_{2}} \sum_{j}\left[\left(s_{j}-\hat{s}\left(x_{j}\right)\right)^{m}-a_{2}-b_{2}\left(\ln (x)-\ln \left(x_{j}\right)\right)\right]^{2} k\left(\frac{\ln \left(x_{j}\right)-\ln (x)}{h}\right)
$$

This gives us the estimator $\hat{\mu}^{m}(x)$. Using a proof similar to the one of Fan and Yao (1998, Theorem 1) it can be shown that the subsitution of $\bar{s}\left(x_{j}\right)$ by the estimator $\hat{s}\left(x_{j}\right)$ in (7) does not influence the consistency of $\hat{\mu}^{m}(x)$ nor the asymptotic normality of $\sqrt{n h_{n}}\left(\hat{\mu}^{m}(x)-\mu^{m}(x)\right)$.

Observe that our estimation procedure assumes that expenditures are independently distributed from the heterogeneity factor. It would be possible to extend our results to allow for endogeneity of the total expenditure by using nonparametric IV or control function methods for regression models under endogeneity. We refer to Imbens and Newey (2009) and Kasy (2014) for possible approaches. A particular difficulty, however, lies in finding an appropriate instrument. 
Testing the HMUI assumption For fixed numbers $I, K, J \in \mathbb{N}$, consider a finite set of income levels $\left[y^{1}, \ldots, y^{I}\right]$ a finite set of values $\left[\pi^{1}, \ldots, \pi^{K}\right]$ from $(0,1)$ and a finite number of integers $\left[m^{1}, \ldots, m^{J}\right]$ larger than 1 . Let $x$ be some fixed income level and define

$$
\begin{aligned}
\theta_{\pi^{k}}\left(y^{i}\right) & =\frac{z_{\pi^{k}}(x) x}{z_{\pi^{k}}\left(y^{i}\right) y^{i}}, \\
\tau_{m^{j}}\left(y^{i}\right) & =\left(\frac{\mu^{m^{j}}(x)}{\mu^{m^{j}}\left(y^{i}\right)}\right)^{1 / m^{j}} \frac{x}{y^{i}} .
\end{aligned}
$$

Conditions (3) and (5) show that if the HMUI assumption holds and if all moments $\mu^{m^{j}}$ (.) exist, then the values of $\theta_{\pi^{k}}\left(y^{i}\right)$ and $\tau_{m^{j}}\left(y^{i}\right)$ should be equal for all values of $\pi^{k}$ and $m^{j}$. In other words, for all $y^{i}, \pi^{\ell}, \pi^{k}, m^{j}$ and $m^{o}$,

$$
\theta_{\pi^{j}}\left(y^{i}\right)=\theta_{\pi^{k}}\left(y^{i}\right)=\tau_{m^{j}}\left(y^{i}\right)=\tau_{m^{o}}\left(y^{i}\right) .
$$

let,

$$
Z=\max _{\left\{y^{i}\right\}_{i \leq I}}\left\{\max _{\left\{m^{j}\right\}_{j \leq J},\left\{\pi^{k}\right\}_{k \leq K}}\left\{\theta_{\pi^{k}}\left(y^{i}\right), \tau_{m^{j}}\left(y^{i}\right)\right\}-\min _{\left\{m^{j}\right\}_{j \leq J},\left\{\pi^{k}\right\}_{k \leq K}}\left\{\theta_{\pi^{k}}\left(y^{i}\right), \tau_{m^{j}}\left(y^{i}\right)\right\}\right\} .
$$

Observe that by definition $Z \geq 0$. However, if the HMUI hypothesis holds, then $Z=0$. As such, we can test the HMUI by developing a test for the nulhypothesis $Z=0$ against the alternative hypothesis that $Z>0$. Consider the estimate $\hat{Z}_{n}$ of $Z$ obtained by replacing the quantiles $z_{\pi}($. and moments $\mu^{m}($.$) by their estimates \hat{z}_{\pi}($.$) and \hat{\mu}^{m}($.$) using a sample of size n$ and a bandwidth of size $h_{n}$. Given the finiteness of $I, K$ and $J$, we know that the random variable $\sqrt{n h_{n}}\left(\hat{Z}_{n}-Z\right)$ has a non-degenerate limiting distribution. A one-sided $(1-\alpha) \%$ confidence interval for this random variable is determined by the value $c_{\alpha}$ for which,

$$
\begin{aligned}
& \operatorname{Pr}\left(\sqrt{n h_{n}}\left(\hat{Z}_{n}-Z\right) \leq c_{\alpha}\right)=1-\alpha, \\
\Longleftrightarrow & \operatorname{Pr}\left(Z \geq-\frac{c_{\alpha}}{\sqrt{n h_{n}}}+\hat{Z}_{n}\right)=1-\alpha .
\end{aligned}
$$

In order to test the nulhypothesis, we find the number $c_{\alpha}$, compute the value of $\frac{-c_{\alpha}}{\sqrt{n h_{n}}}+\hat{Z}_{n}$ and verify if this value is larger than zero. We reject the HMUI assumption at the $(1-\alpha) \%$ confidence level if it is.

The (asymptotic) distribution of $\sqrt{n h_{n}}\left(\hat{Z}_{n}-Z\right)$ is not known. In addition, a bootstrap approximation would not be valid given that the definition of $Z$ contains the max and min functions (see Andrews (2000) for a discussion). Moreover, kernel estimators have an asymptotic bias, which further complicates the bootstrap procedure (see, for example Horowitz (2001)). As a solution, we resort to a subsampling procedure. Although subsampling underperforms the bootstrap in settings where both are applicable, it has the advantage that it is valid under very weak conditions. We refer to Politis, Romano, and Wolf (1999) for a detailed discussion of this procedure. 
The idea of subsampling is to take subsamples from the observed sample of size $w \ll n$ without replacement. Assume that the corresponding bandwidth is given by $h_{w}$ with $h_{w} \rightarrow 0$ and $w h_{w} \rightarrow$ $\infty$ and let $Z_{w}^{*}$ be the estimate of $Z$ obtained from such a subsample. If we take a large number of subsamples, we can compute the value of $\hat{c}_{\alpha}$ such that for $(1-\alpha) 100 \%$ of the subsamples,

$$
\sqrt{w h_{w}}\left(Z_{w}^{*}-\hat{Z}_{n}\right) \leq \hat{c}_{\alpha}
$$

Then asymptotically, for $w h_{w} \rightarrow \infty$ and $n h_{n} /\left(w h_{w}\right) \rightarrow \infty$,

$$
\operatorname{Pr}\left(\sqrt{n h_{n}}\left(\hat{Z}_{n}-Z\right) \leq \hat{c}_{\alpha}\right) \rightarrow(1-\alpha)
$$

This gives the following procedure. First, we take a large number of subsamples of size $w$ and compute the value of $\hat{c}_{\alpha}{ }^{4}$ Next, we compute $\frac{-\hat{c}_{\alpha}}{\sqrt{n h_{n}}}+\hat{Z}_{n, h}$ and look whether this value is larger than zero. If it is, then we reject the HMUI assumption at the $(1-\alpha) \%$ confidence level.

\section{Application}

Data description We use data from the Consumer Expenditure survey data set (CEX) which is a US consumer cross sectional budget survey. We focus on the 2007 diary survey and we base our analysis on the consumption share of food. ${ }^{5}$ Taking food as the commodity of interest has the advantage that it is non-durable. As the diary survey reports expenditures on a two-week basis, we first convert these to yearly equivalents. Next, we deseasonalize using a dummy regression approach. ${ }^{6}$ In order to take into account that variation in expenditures can be driven by the household composition, e.g. the number of adults or the number of kids living in the family we deflate total expenditures by the OECD equivalence scale of the household. Given that we use the diary data, the expenditure level used should be interpreted as expenditures on nondurable consumption. The restriction to nondurables is valid if nondurable consumption is separable in the utility function.

Next, we control for several observable characteristics by restricting our sample to (i) households who have completed the two-week diary, (ii) households who are not living in student housing, (iii) households who are vehicle owners, (iv) households where both members work at least 17 hours, (v) households in which both members are not self-employed, (vi) households in which the age of the reference person is at least 21 and finally we restrict attention to (vii) households that consist of a husband, a wife and possibly children. Finally, we also remove some outlier observations. ${ }^{7}$ This leaves us with a sample size of 2163 observations.

\footnotetext{
${ }^{4}$ We draw 10.000 subsamples of size $m=n^{0.7}$. Results are not sensitive to variations in this subsample size.

${ }^{5}$ Food is an aggregate of cereals, bakery products, beef, pork, poultry, seafood, other meat, eggs, milk products, other dairy products, fresh fruit, fresh vegetables, processed fruit, processed vegetables, sweets, fat and oils, nonalcoholic beverages, prepared food, snacks and condiments.

${ }^{6}$ Specifically, the expenditures on each category (reported for two weeks) are regressed on month dummies. Residuals from this regression (which can be interpreted as the variation in expenditures which can not be explained by seasonality or by months) are added to the mean expenditures for each category in order to construct deseasonalized expenditures.

${ }^{7}$ In particular, we removed observations for which rescaled total expenditures or expenditure shares are not within
} 
Figure 1: Estimates of the marginal utility of income $\left(\hat{\theta}_{\pi}\left(y^{i}\right)\right)$ left and $\tau_{m}\left(y^{i}\right)$ right
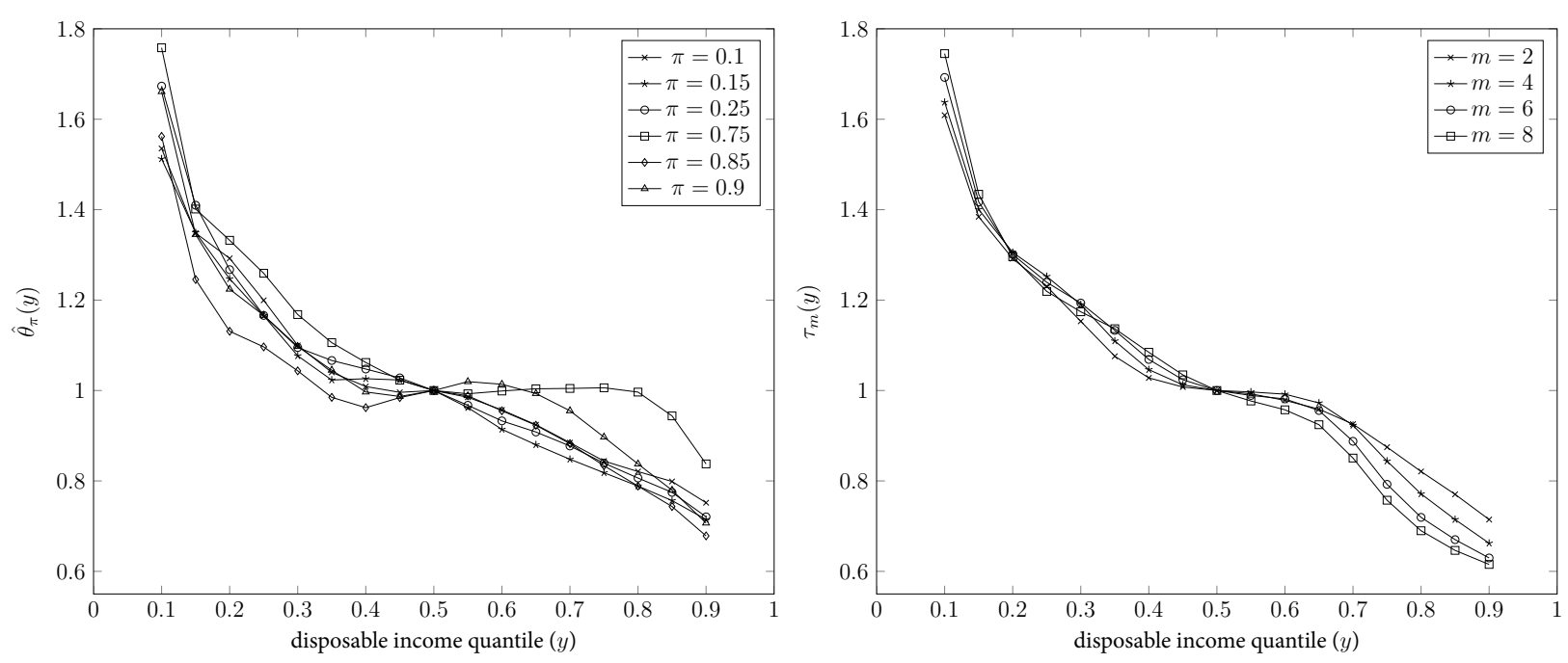

Results Figure 1 plots the values of $\hat{\theta}_{\pi}(y)=\frac{\hat{z}_{\pi}(x) x}{\hat{z}_{\pi}(y) y}$ and $\hat{\tau}_{m}(y)=\left(\frac{\hat{\mu}^{m}(x)}{\hat{\mu}^{m}(y)}\right)^{1 / m} \frac{x}{y}$ where $x$ is the median expenditure level in 2007 and $y$ ranges over the different quantiles of the expenditure level in the data set. We focus on the values of $\pi=0.1,0.15,0.25,0.75,0.85$ and 0.9 which are sufficiently far away from the average in the sample such that $z_{\pi}(y) \neq 0$ and on the even moments $m=2,4,6,8$. Tables 1 and 2 in Appendix A contain the exact figures. If the HMUI hypothesis holds, then these graphs should trace out the marginal utility of income function $\beta(y) / \beta(x)$ for varying value of $y$. We see that in most cases the graphs are downward sloping, which is consistent with the widely accepted idea that the marginal utility of income is decreasing, i.e. one additional dollar is worth more to a poor person than to a rich person. The HMUI hypothesis holds if these various graphs are identical for varying values of $\pi$ and $m$. Visual inspection shows that the graphs are indeed quite close to each other. Implementing our test gives for a significance level of $\alpha=$ 0.05 a critical value (i.e. $-\hat{c}_{\alpha} / \sqrt{n h}+\hat{Z}_{n, h}$ ) of -0.1138 and a critical value of -0.0354 for a significance level of $\alpha=0.10$. The $p$-value for the hypothesis that $Z=0$ is equal to 0.1364 . As such, on the basis of our test, we do not reject the hypothesis that $Z=0$ at the $90 \%$ confidence level.

Figure 2 and Table 1 give the estimate of the marginal utility of income function $\frac{\beta(y)}{\beta(x)}$ obtained as the average over all considered quantiles and moments together with the $95 \%$ pointwise CI based on the same subsampling procedure. The marginal utility of income function allows us to make interpersonal welfare comparisons. For example, redistributing one dollar from an individual at the median income level towards an individual at the first decile improves aggregate utility by $1.64-1=0.64$. In other words, in order to increase aggregate utility by one unit, one can

3 standard deviations from the mean and observations for which rescaled total expenditures are among the 5 per cent lowest or 5 per cent highest expenditures or for which the expenditure share is close to 0 . 
either allocate $1 \$$ to somebody at the median income level or $0.61 \$$ (i.e. $1 / 1.64$ ) to somebody at the first decile. Giving the same dollar to someone at the 9th decile only increases aggregate utility by 0.69 . Alternatively, in order to increase utility by one unit, one should allocate as much as $1.45 \$$ (i.e. $1 / 0.69$ ) to somebody at the 9 th decile.

Figure 2: Estimates of the marginal utility of income with $90 \%$ confidence interval

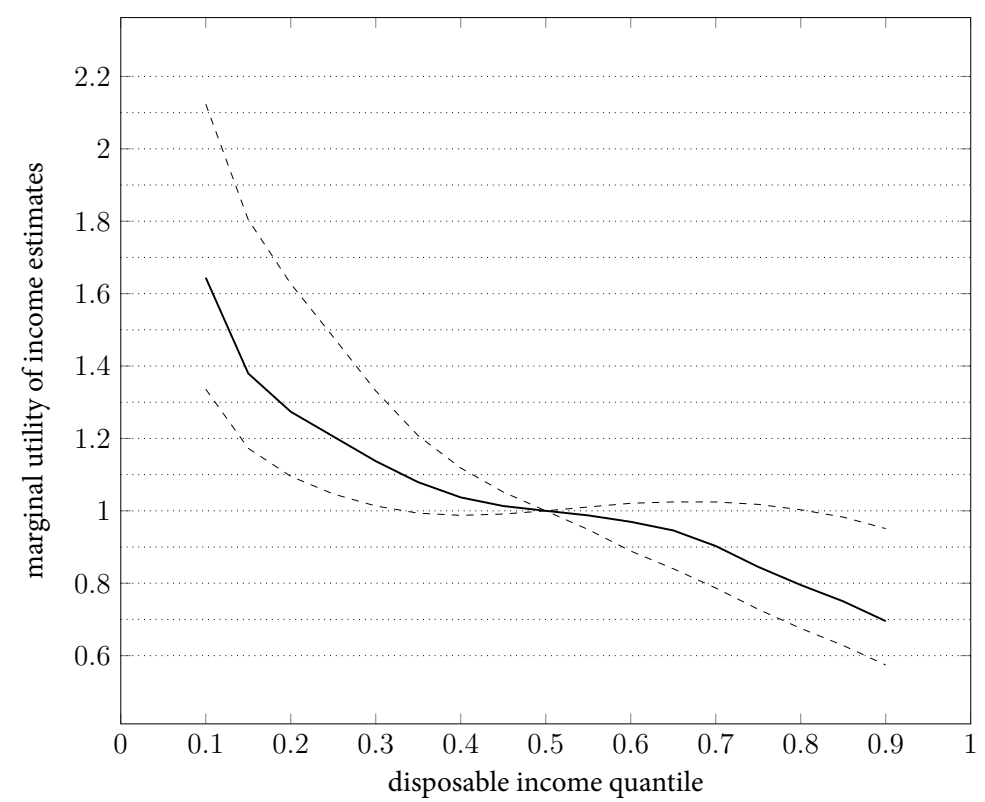

\section{Conclusion}

We developed a test to verify whether a population of individuals have the same marginal utility of income function. The homogeneous marginal utility of income assumption is frequently used in applied demand and welfare analyses. In addition, if the test is not rejected, it allows us to identify the marginal utility of income function. Interestingly, our test and identification results are modest in terms of data requirements. We apply our procedure to a US cross sectional data set on food consumption and we find that can not reject the homogeneous marginal utility of income hypothesis at the $90 \%$ confidence level. We estimate that one dollar is more than twice as valuable to someone at the first decile compared to someone at the ninth decile of the (disposable) income distribution.

\section{References}

Andrews, D. W. K., 2000. Inconsistency of the bootstrap when a parameter is on the bound of the parameter space. Econometrica 68, 399-405. 
Blundell, R., Browning, M., Crawford, I., 2003. Nonparametric engel curves and revealed preference. Econometrica 71, 205-240.

Blundell, R., Horowitz, J. L., Parey, M., 2012. Measuring the price responsiveness of gasoline demand: Economic shape restrictions and nonparametric demand estimation. Quantitative Economics 3, 29-51.

Blundell, R., Kristensen, D., Matzkin, R., 2014. Bounding quantile demand functions using revealed preference inequalities. Journal of Econometrics 179, 112-127.

Deaton, A., Muellbauer, J., 1980. Economics and Consumer Behavior. Cambridge University Press.

Fan, J., Yao, Q., 1998. Efficient estimation of conditional variance functions in stochastic regressions. Biometrika 85 (645-660).

Frisch, R., 1932. New Methods for Measuring Marginal Utility.

Gorman, W. M., 1961. On a class of preference fields. Metroeconomica 13, 53-56.

Hausman, J. A., Newey, W. K., 1995. Nonparametric estimation of exact consumers surplus and deadweight loss. Econometrica 63, 1445-1476.

Hausman, J. A., Newey, W. K., 2013. Individual heterogeneity and average welfare. Tech. rep., cemmap.

Horowitz, J. L., 2001. Handbook of Econometrics, edition 1. Elsevier, Ch. The Bootstrap, pp. 3159-3228.

Imbens, G. W., Newey, W. K., 2009. Identification and estimation of triangular simultaneous equations models without additivity. Econometrica 77, 1481-1512.

Kasy, M., 2014. Instrumental variables with unrestricted heterogeneity and continuous treatment. The Review of Economic Studies forthcoming.

Layard, R., Nickell, S., Mayraz, G., 2008. The marginal utility of income. Journal of Public Economics 92, 1846-1857.

Lewbel, A., 2001. Demand systems with and without errors. American Economic Review 91, 611-618.

Li, Q., Racine, S., 2007. Nonparametric Econometrics. Princeton University Press, Princeton.

Morgan, J. N., 1945. Can we measure the marginal utility of money? Econometrica 13, 129-152.

Politis, D. N., Romano, J. P., Wolf, M., 1999. Subsampling. Springer.

Samuelson, P. A., 1937. A note on measurement of utility. The Review of Economic Studies 4, $155-161$. 
Schlee, E. E., 2007. Measuring consumer welfare with mean demands. International Economic Review.

Vickrey, W., 1945. Measuring marginal utility by reactions to risk. Econometrica 13, 319-333.

Zhou, T., Zhu, L., 2014. Conditional median absolute deviation. Journal of Statistical Computation and Simulation forthcoming.

\section{A Tables}


Table 1: Estimates of $\beta(y) / \beta(x)$ using the conditional quantiles

\begin{tabular}{ccccccc}
\hline percentile & $\pi=0.1$ & $\pi=0.15$ & $\pi=0.25$ & $\pi=0.75$ & $\pi=0.85$ & $\pi=0.9$ \\
& & & & & & \\
\hline 0.10 & 1.5353 & 1.5125 & 1.6733 & 1.7581 & 1.5621 & 1.6611 \\
0.15 & 1.3481 & 1.3493 & 1.4099 & 1.4014 & 1.2456 & 1.3451 \\
0.20 & 1.2923 & 1.2470 & 1.2673 & 1.3322 & 1.1311 & 1.2242 \\
0.25 & 1.1996 & 1.1655 & 1.1662 & 1.2594 & 1.0964 & 1.1662 \\
0.30 & 1.0997 & 1.0767 & 1.0951 & 1.1680 & 1.0435 & 1.0974 \\
0.35 & 1.0402 & 1.0232 & 1.0664 & 1.1059 & 0.9848 & 1.0449 \\
0.40 & 1.0088 & 1.0261 & 1.0475 & 1.0620 & 0.9621 & 0.9968 \\
0.45 & 0.9960 & 1.0233 & 1.0279 & 1.0228 & 0.9843 & 0.9869 \\
0.50 & 1.0000 & 1.0000 & 1.0000 & 1.0000 & 1.0000 & 1.0000 \\
0.55 & 0.9851 & 0.9622 & 0.9673 & 0.9928 & 0.9883 & 1.0197 \\
0.60 & 0.9570 & 0.9142 & 0.9328 & 0.9989 & 0.9552 & 1.0138 \\
0.65 & 0.9243 & 0.8800 & 0.9082 & 1.0038 & 0.9231 & 0.9933 \\
0.70 & 0.8846 & 0.8479 & 0.8773 & 1.0046 & 0.8825 & 0.9552 \\
0.75 & 0.8440 & 0.8182 & 0.8394 & 1.0061 & 0.8337 & 0.8970 \\
0.80 & 0.8208 & 0.7887 & 0.8064 & 0.9965 & 0.7887 & 0.8375 \\
0.85 & 0.7988 & 0.7566 & 0.7747 & 0.9439 & 0.7434 & 0.7795 \\
0.90 & 0.7518 & 0.7149 & 0.7206 & 0.8377 & 0.6785 & 0.7069 \\
\hline
\end{tabular}


Table 2: Estimates of $\beta(y) / \beta(x)$ using the conditional moments percentile $m=2 \quad m=4 \quad m=6 \quad m=8$

\begin{tabular}{lllll}
\hline 0.10 & 1.6090 & 1.6376 & 1.6925 & 1.7452 \\
0.15 & 1.3842 & 1.4005 & 1.4172 & 1.4341 \\
0.20 & 1.2917 & 1.3055 & 1.3009 & 1.2960 \\
0.25 & 1.2304 & 1.2523 & 1.2394 & 1.2192 \\
0.30 & 1.1530 & 1.1901 & 1.1935 & 1.1741 \\
0.35 & 1.0758 & 1.1097 & 1.1331 & 1.1367 \\
0.40 & 1.0279 & 1.0462 & 1.0687 & 1.0845 \\
0.45 & 1.0077 & 1.0125 & 1.0238 & 1.0346 \\
0.50 & 1.0000 & 1.0000 & 1.0000 & 1.0000 \\
0.55 & 0.9927 & 0.9968 & 0.9891 & 0.9768 \\
0.60 & 0.9782 & 0.9919 & 0.9814 & 0.9573 \\
0.65 & 0.9588 & 0.9725 & 0.9558 & 0.9244 \\
0.70 & 0.9257 & 0.9230 & 0.8877 & 0.8506 \\
0.75 & 0.8747 & 0.8439 & 0.7925 & 0.7574 \\
0.80 & 0.8209 & 0.7714 & 0.7195 & 0.6899 \\
0.85 & 0.7704 & 0.7147 & 0.6702 & 0.6464 \\
0.90 & 0.7146 & 0.6623 & 0.6297 & 0.6154 \\
\hline
\end{tabular}


Table 3: Estimates of $\beta(y) / \beta(x)$ with the $90 \%$ confidence bounds percentile lowerbound estimate upperbound

\begin{tabular}{llll}
\hline 0.10 & 1.3361 & 1.6440 & 2.1235 \\
0.15 & 1.1726 & 1.3794 & 1.8040 \\
0.20 & 1.0952 & 1.2737 & 1.6274 \\
0.25 & 1.0466 & 1.2054 & 1.4803 \\
0.30 & 1.0134 & 1.1372 & 1.3313 \\
0.35 & 0.9935 & 1.0790 & 1.2073 \\
0.40 & 0.9875 & 1.0370 & 1.1184 \\
0.45 & 0.9911 & 1.0133 & 1.0520 \\
0.50 & 1.0000 & 1.0000 & 1.0000 \\
0.55 & 0.9483 & 0.9874 & 1.0106 \\
0.60 & 0.8888 & 0.9696 & 1.0208 \\
0.65 & 0.8400 & 0.9458 & 1.0246 \\
0.70 & 0.7868 & 0.9027 & 1.0245 \\
0.75 & 0.7280 & 0.8451 & 1.0177 \\
0.80 & 0.6752 & 0.7951 & 1.0028 \\
0.85 & 0.6280 & 0.7499 & 0.9827 \\
0.90 & 0.5740 & 0.6953 & 0.9509 \\
\hline
\end{tabular}

